University of Nebraska - Lincoln

DigitalCommons@University of Nebraska - Lincoln

Electron self-injection into an evolving plasma bubble: Quasimonoenergetic laser-plasma acceleration in the blowout regime

Serguei Y. Kalmykov

University of Nebraska-Lincoln, s.kalmykov.2013@ieee.org

A. Beck

CEA, DAM, DIF, Arpajon F-91297, France

S. A. Yi

University of Texas at Austin

V. N. Khudik

University of Texas at Austin

Michael C. Downer

University of Texas at Austin, downer@physics.utexas.edu

See next page for additional authors

Follow this and additional works at: https://digitalcommons.unl.edu/physicsumstadter

Part of the Physics Commons

Kalmykov, Serguei Y.; Beck, A.; Yi, S. A.; Khudik, V. N.; Downer, Michael C.; Lefebvre, E.; Shadwick, Bradley Allan; and Umstadter, Donald, "Electron self-injection into an evolving plasma bubble: Quasimonoenergetic laser-plasma acceleration in the blowout regime" (2011). Donald Umstadter Publications. 81.

https://digitalcommons.unl.edu/physicsumstadter/81

This Article is brought to you for free and open access by the Research Papers in Physics and Astronomy at DigitalCommons@University of Nebraska - Lincoln. It has been accepted for inclusion in Donald Umstadter Publications by an authorized administrator of DigitalCommons@University of Nebraska - Lincoln. 


\section{Authors}

Serguei Y. Kalmykov, A. Beck, S. A. Yi, V. N. Khudik, Michael C. Downer, E. Lefebvre, Bradley Allan Shadwick, and Donald Umstadter 


\title{
Electron self-injection into an evolving plasma bubble: Quasi-monoenergetic laser-plasma acceleration in the blowout regime ${ }^{a)}$
}

\author{
S. Y. Kalmykov, ${ }^{1, b), c)}$ A. Beck, ${ }^{2}$ S. A. Yi, ${ }^{3}$ V. N. Khudik, ${ }^{3}$ M. C. Downer, ${ }^{3}$ E. Lefebvre, ${ }^{2}$ \\ B. A. Shadwick, ${ }^{1}$ and D. P. Umstadter ${ }^{1}$ \\ ${ }^{1}$ Department of Physics and Astronomy, University of Nebraska - Lincoln, Lincoln, Nebraska 68588-0299, \\ USA \\ ${ }^{2}$ CEA, DAM, DIF, Arpajon F-91297, France \\ ${ }^{3}$ Department of Physics, C1500, The University of Texas at Austin, Austin, Texas 78712, USA
}

(Received 26 November 2010; accepted 27 January 2011; published online 12 April 2011)

\begin{abstract}
An electron density bubble driven in a rarefied uniform plasma by a slowly evolving laser pulse goes through periods of adiabatically slow expansions and contractions. Bubble expansion causes robust self-injection of initially quiescent plasma electrons, whereas stabilization and contraction terminate self-injection thus limiting injected charge; concomitant phase space rotation reduces the bunch energy spread. In regimes relevant to experiments with hundred terawatt- to petawatt-class lasers, bubble dynamics and, hence, the self-injection process are governed primarily by the driver evolution. Collective transverse fields of the trapped electron bunch reduce the accelerating gradient and slow down phase space rotation. Bubble expansion followed by stabilization and contraction suppresses the low-energy background and creates a collimated quasi-monoenergetic electron bunch long before dephasing. Nonlinear evolution of the laser pulse (spot size oscillations, self-compression, and front steepening) can also cause continuous self-injection, resulting in a large dark current, degrading the electron beam quality. (c) 2011 American Institute of Physics. [doi:10.1063/1.3566062]
\end{abstract}

\section{INTRODUCTION}

Progress in ultrahigh-power, short-pulse laser technology made it possible to realize the blowout regime $e^{1-7}$ of laserplasma electron acceleration (LPA) in the laboratory ${ }^{8-10}$ and produce first gigaelectron volt-class beams from centimeterlength gaseous plasmas. ${ }^{11-15}$ Both numerical modeling ${ }^{3,16-18}$ and direct experimental diagnostics ${ }^{19,20}$ show close correlation between generation of collimated, quasi-monoenergetic electron beams and formation of a unique plasma structureelectron density "bubble" - trailing in the wake of a tightly focused ultraintense laser pulse $\left(I_{\text {peak }}>10^{19} \mathrm{~W} / \mathrm{cm}^{2}\right)$. The laser ponderomotive force creates full electron cavitation behind the driver, while fully stripped ions remain immobile. Fields due to this charge separation attract bulk electrons to the axis, and their trajectories overshoot. The resulting closed cavity of electron density, surrounded by a dense shell (sheath) of relativistic electrons, propagates with a near-luminous speed over a positive ion background and guides the laser pulse over many Rayleigh lengths until depletion. ${ }^{6}$ The Lorentz factor associated with the pulse group velocity, $\gamma_{g} \approx \omega_{0} / \omega_{p e}$, is about 20 in current experiments and shall approach 100 in near-term experiments with short-pulse petawatt lasers ${ }^{17,18}$ (here, $\omega_{0}$ is the laser frequency, $\omega_{p e}=\left(4 \pi e^{2}\right.$ $\left.n_{0} / m_{e}\right)^{1 / 2}$ is the Langmuir electron frequency, $m_{e}$ is the electron rest mass, $n_{0}$ is the background electron density, and $e$ is the electron charge). Slow evolution of the self-guided pulse causes variations of the bubble shape and wake potentials. As a result of this evolution, sheath electrons can penetrate into

\footnotetext{
a) Paper KI3 3, Bull. Am. Phys. Soc. 55, 190 (2010).

b) Invited speaker.

${ }^{\text {c)} E l e c t r o n i c ~ a d d r e s s: ~ s k a l m y k o v 2 @ u n l . e d u . ~}$
}

the bubble near its rear, synchronize with it (i.e., obtain the longitudinal momentum $p_{\|} \approx \gamma_{g} m_{e} c$ ) and then travel inside the cavity, continuously gaining energy. ${ }^{18,21-27}$ Self-injection eliminates the need for an external injector and, thus, is favorable for the accelerator design. It ties the electron beam quality to the self-consistent nonlinear evolution of the driver. ${ }^{18,27}$ Experiments also demonstrate that even in high-density plasmas, such as $\gamma_{g}<25$, bubble formation alone is not sufficient for self-injection and production of high-energy electrons. ${ }^{13,14,19,20}$ Therefore, understanding the self-injection process and its relation to nonlinear relativistic optical dynamics of the driver is vital for the production of high-quality beams.

The importance of driver evolution for initiation and termination of self-injection was recognized quite early. ${ }^{21}$ It has also been understood ${ }^{26,27}$ that laser diffraction followed by self-guiding is the most attractive scenario for the formation of a monoenergetic electron beam. As an initially overfocused laser diffracts, the bubble expands, and electrons are injected continuously. When self-guiding sets in, the bubble stabilizes, and self-injection terminates. Electrons injected during the expansion travel deep inside the bucket and are continuously accelerated. At the same time, electrons injected immediately before the expansion stops are located in the region of the highest accelerating gradient. They rapidly equalize in energy with earlier injected particles, and a monoenergetic bunch forms long before dephasing. ${ }^{24,26,27}$ Secondary injection into the same bucket (dark current) remains suppressed, and low-energy tails ${ }^{12-14,28,29}$ do not develop in the electron spectra.

Current experiments are not optimized for the above scenario. In most cases, the pulse is strongly overcritical, 
$P \gtrsim 10 P_{\text {cr }}$ (where $P_{\mathrm{cr}}=16.2 \gamma_{g}^{2} \mathrm{GW}$ is the critical power for the relativistic self-focusing ${ }^{30}$ ), and its length is close to the plasma period. The spot size of such pulse oscillates during self-guiding, self-injection resumes periodically, and electron beam emittance grows. ${ }^{17,18,22,25}$ Besides, pulse self-compression often leads to continuous injection that eventually overloads the bubble and results in poor beam collimation. $^{12,13}$ The leading edge of the pulse witnesses a nonlinear index down-ramp at all times, which causes localized frequency redshifting and bandwidth increase. Group velocity dispersion concurrently compresses the pulse. ${ }^{31-33}$ Concomitant depletion of the leading edge further enhances pulse self-steepening. ${ }^{6,34}$ The initially smooth driver turns into a relativistically intense "piston," which preaccelerates and compresses the initially quiescent electron fluid by its steep leading edge. The large charge separation immediately behind the driver results in sheath electrons receiving strong longitudinal kick, increasing their inertia and delaying their return to the axis. As a result, the bubble elongates, and massive, uninterrupted self-injection follows. We find that, in spite of high injected charge, this scenario remains the same in both quasistatic and fully explicit electromagnetic threedimensional particle-in-cell (3D PIC) simulations. Beam loading ${ }^{35}$ becomes important only in the final stage of this process. Importantly, transverse matching of the pulse for self-guiding precludes neither periodic nor continuous injection. ${ }^{17}$ Emittance growth due to continuous injection is a serious concern for such demanding applications as compact hard x-ray sources. ${ }^{36,37}$

In this paper, we examine different scenarios of electron self-injection in a single numerical experiment. Two complementary simulation approaches are used. In Sec. II, we elucidate the physics and develop the conceptual framework of the problem using the quasistatic, cylindrically symmetric, fully relativistic PIC code WAKE. ${ }^{38}$ A fully $3 \mathrm{D}$, nonaveraged, dynamic test electron tracking module incorporated in WAKE (Refs. 24 and 39) emulates the nonquasistatic response of initially quiescent electrons to a high-frequency quasiparaxial laser field and slowly varying electromagnetic plasma wakes. In Sec. III, we validate the test-particle results in a full 3D PIC simulation using the code CALDERCIRC. $^{40}$

The formation of a quasi-monoenergetic electron bunch during one period of laser spot oscillation is the subject of Secs. II A and II B. By analyzing quasistatic trajectories and using the results of test electron tracking (Sec. II A), we identify precisely the injection candidates, collection volume, and evaluate the minimal bubble expansion rate for the initiation of self-injection. Results of Sec. II B show that self-injection and subsequent acceleration of an electron require initial reduction of its moving-frame (MF) Hamiltonian. Laser pulse self-compression and resulting continuous injection are considered in Sec. II C. In Sec. III, we validate the self-injection scenarios discussed in Sec. II in a CALDERCIRC simulation. We find that the test-particle modeling correctly identifies the physical processes responsible for the initiation and termination of self-injection. In Sec. IV, we summarize our results and point out directions of our future work.

\section{SCENARIOS OF SELF-INJECTION IN THE BLOWOUT REGIME}

For a standard set of parameters of LPA experiments at the University of Nebraska, ${ }^{41}$ we examine various scenarios of electron self-injection and relate these scenarios to nonlinear dynamical processes involving the laser pulse. A transform-limited, linearly polarized Gaussian laser pulse with a full width at half-maximum in intensity $\tau_{\mathrm{L}}=30 \mathrm{fs}$ and central wavelength $\lambda_{0}=0.805 \mu \mathrm{m}$ is focused at the plasma border $(z=0)$ to a spot size $r_{0}=13.6 \mu \mathrm{m}$ corresponding to a Rayleigh length of $0.72 \mathrm{~mm}$. The plasma has a $0.5 \mathrm{~mm}$ linear entrance ramp followed by $1.7 \mathrm{~mm}$ plateau of density $n_{0}=6.5 \times 10^{18} \mathrm{~cm}^{-3}\left(\gamma_{g}=16.3, \omega_{p e} \tau_{\mathrm{L}}=4.3\right)$. The laser power is $70 \mathrm{TW}$, which yields $P / P_{\mathrm{cr}}=16.25$, a peak intensity at the focus $I_{\text {peak }}=2.3 \times 10^{19} \mathrm{~W} / \mathrm{cm}^{2}$, and normalized vector potential $a_{0}=3.27$.

The quasistatic nature of the bulk plasma response makes it possible to elucidate the physics of self-injection using a conceptually simple and computationally efficient toolbox: a fully relativistic $3 \mathrm{D}$ particle tracking module ${ }^{24,39}$ built into the cylindrically symmetric time-averaged quasistatic PIC code WAKE. ${ }^{38}$ WAKE models the laser pulse propagation using an extended paraxial solver. It preserves the group velocity dispersion in the vicinity of the carrier frequency and calculates precisely radiation absorption due to the wake excitation. The test-particle module is fully dynamic, making no assumption of cylindrical symmetry, and is not time-averaged. In particular, it takes into account the interaction of test electrons with the nonaveraged, linearly polarized laser field with nonparaxial corrections. ${ }^{39,42}$ To capture the laser pulse interaction with nonquasistatic background electrons (and thus to model self-injection into nonstationary quasistatic wake fields), a group of quiescent test electrons is placed before the laser pulse at each time step. In this way, electron self-injection associated with bubble and driver evolution is separated from the effects brought about by the collective fields of the trapped electron bunch, i.e., from effects due to beam loading. ${ }^{35}$ This simulation approach allows to fully characterize details of the self-injection process ${ }^{18,26,27}$ and to relate the injection process to dynamics of the laser and the bubble using a nonstationary Hamiltonian formalism. ${ }^{24,26}$ The WAKE simulation uses the grid $d \xi=0.035 k_{p}^{-1} \approx 0.073 \mu \mathrm{m}, d r \approx 0.1 k_{p}^{-1}$ with 30 macroparticles per radial cell, and time step $d t=d z / c$ $\approx 1.325 \omega_{0}^{-1}$. Here, $\xi=z-c t$ and $k_{p}=\omega_{p e} / c$.

\section{A. Injection candidates, collection volume, and minimal expansion rate to initiate self-injection}

The laser pulse self-focuses upon entering the plasma and reaches the highest intensity at $z \approx 0.8 \mathrm{~mm}$. Almost complete blowout is maintained over the entire propagation distance. As seen in Fig. 1, bubble expansion and electron injection begin soon after the laser pulse enters the density plateau. Figure 2 shows a fully expanded bubble at $z=1.04 \mathrm{~mm}$. Macroparticles whose trajectories are shown in Fig. 2(a) obey the quasistatic approximation and, thus, cannot be trapped. However, analysis of quasistatic electron flow helps to identify injection candidates and specify the scenario of bubble 

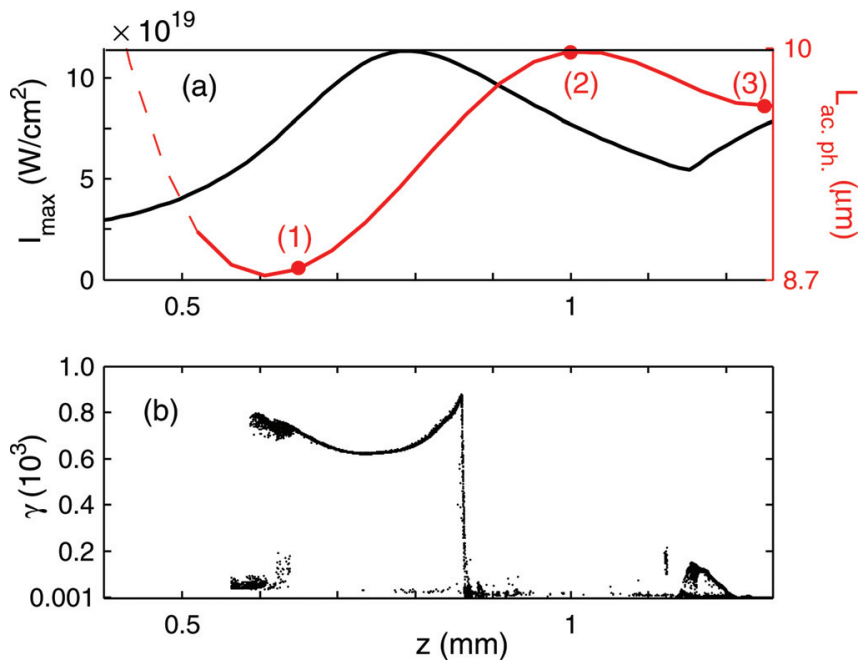

FIG. 1. (Color online) (a) Peak laser intensity (black) and length of the accelerating phase [red (gray)] vs propagation distance. Data from positions (1) to (3) are used to describe the process of monoenergetic electron bunch formation in Fig. 3. (b) Energy of test electrons vs their initial positions after one period of bubble size oscillation [position (3)]. All electrons accelerated beyond $100 \mathrm{MeV}$ were collected during the interval of bubble expansion.

evolution favorable for injection. This analysis also provides precise estimates of the collection volume and the bubble expansion rate necessary to initiate the injection. Each macroparticle can be put into one of three clearly defined groups
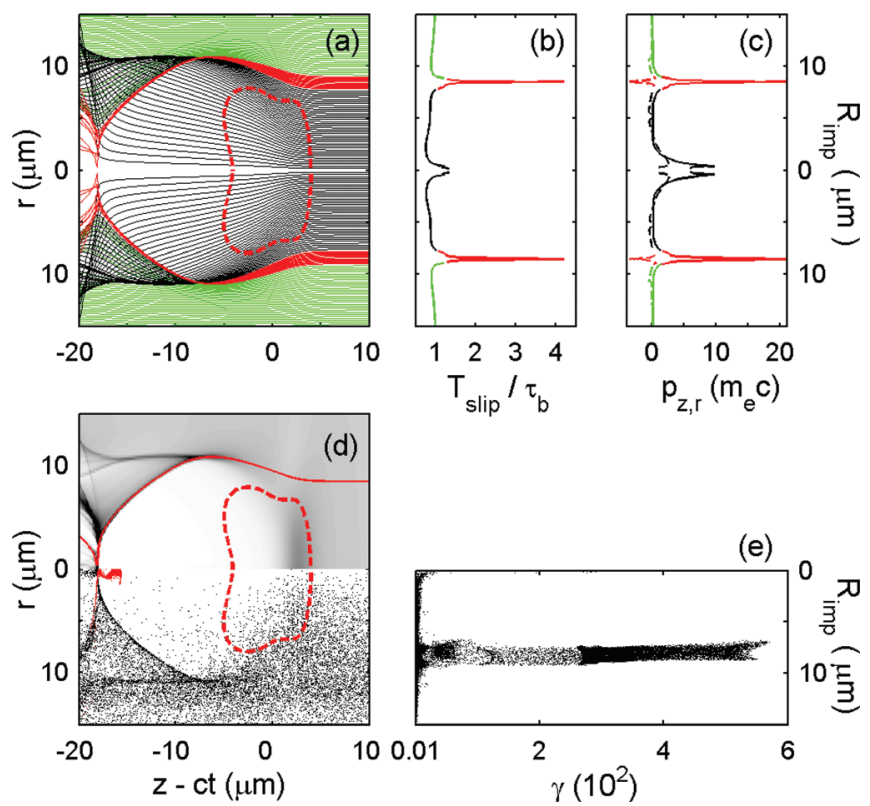

FIG. 2. (Color online) Fully expanded electron bubble from the waKe simulation. Dashed red (dark gray) contour in panels (a) and (d) is the isocontour of laser intensity at $\exp (-2)$ of the peak. (a) Trajectories $r(\xi)$ of the quasistatic macroparticles. Green (light gray) and black trajectories correspond to passing electrons. The red (dark gray) ones correspond to sheath electronsinjection candidates. (b) Normalized slippage time as a function of the impact parameter, $R_{i m p}=r\left(\xi \gg c \tau_{L}\right)$. (c) Longitudinal $\left(p_{z}\right.$, solid line) and transverse $\left(p_{r}\right.$, dashed line) momenta of macroparticles at the rear of the bubble (point of trajectory crossing). Sheath electrons have the largest slippage time and become relativistic before crossing the axis. (d) Top: the quasistatic electron density; grayscale is linear with a cutoff at $n_{e}=3.25 \times 10^{19}$ $\mathrm{cm}^{-3}$. Solid red (dark gray) line is the trajectory of the macroparticle with the greatest slippage time. Bottom: radial positions of nonquasistatic test electrons. Red (dark gray) dots are the electrons with $\gamma>\gamma_{g}=16.3$. (e) Impact parameters of test electrons from panel (d) vs energy. [color coded in Fig. 2(a)]. The majority of electrons, viz., those expelled by the radiation pressure and those attracted from periphery to the axis by the charge separation force, is passing. They fall behind the bubble roughly within a time interval $\tau_{b}=L_{b} / c$, where $L_{b}$ is the bubble length. Sheath electrons are different; they may travel with the bubble over a long distance. Figure 2(b) shows that their slippage time,

$$
T_{\text {slip }}=\int_{0}^{L_{b}} \frac{d \zeta}{c-v_{z}},
$$

significantly exceeds $\tau_{b}$. Here, $\zeta=-\xi$, and $\zeta=L_{b} \approx 18 \mu \mathrm{m}$ is the coordinate of the rear of the bubble. The trajectory of the macroparticle with the largest slippage time, $T_{\text {slip }}$ $\approx 4.2 \tau_{b}$, is shown in Fig. 2(d). Sheath electrons are exposed to the highest wake fields in the system; at the rear of the bubble, they are strongly preaccelerated in both longitudinal and transverse directions: Fig. 2(c) gives $p_{z} /\left(m_{e} c\right) \approx 21$ $>\gamma_{g}$, and $p_{r} \approx-4 m_{e} c$. Their large longitudinal momentum makes these electrons the best injection candidates; their promotion to fully dynamic macroparticles can result in their self-injection and acceleration. ${ }^{43}$ However, these electrons also have relativistic transverse momentum and tend to exit the bubble in the radial direction-such electrons have been earlier observed in the laboratory in the absence of any noticeable trapping. ${ }^{20,44}$ A test particle simulation shows that self-injection into a nonevolving structure, i.e., depending on variables $r$ and $\xi$ only, is highly inefficient. At lower densities, $\gamma_{g} \sim 100$, electrons do not synchronize with the propagating bubble, and self-injection in the nonevolving bubble does not occur. ${ }^{18,26,27}$

To be injected, a sheath electron must be deflected back to axis by the focusing gradient. This gradient is only available inside the cavity. To help the electrons enter into the cavity, the bubble must expand during the slippage time. Energetic sheath electrons can then outrun the boundary of the expanding bubble and stay inside the bubble long enough to both synchronize longitudinally and make a U-turn transversely. The bubble must expand rapidly enough to change its size by an appreciable fraction during the electron transit time $T_{\text {slip. }}$ To separate the most energetic electrons from the sheath, elongation of the bubble over the slippage time has to exceed the thickness of the sheath $\Delta_{\mathrm{sh}}$ at the rear of the bucket ${ }^{26}$ :

$$
\Delta L_{b}=L_{b}\left(t+T_{\text {slip }}\right)-L_{b}(t) \geq \Delta_{\text {sh }}
$$

Indeed, Fig. 2(d) shows a group of particles (red markers) collected by the expanding bubble between $z \approx 0.6 \mathrm{~mm}$ and $z+\Delta z_{\text {exp }} \approx 1 \mathrm{~mm}$. Taking $c T_{\text {slip }} \approx 75 \mu \mathrm{m}$ and using Fig. 1(a) to estimate the full expansion of the bubble, $2 \Delta L_{\text {ac.ph }}$ $\approx 2.6 \mu \mathrm{m}$ (where $L_{\text {ac.ph }}$ is the distance between positions of zero and peak accelerating gradient on axis), we find $\Delta L_{b} \approx\left(2 \Delta L_{\text {ac.ph }} / \Delta z_{\text {exp }}\right) c T_{\text {slip }} \approx 0.5 \mu \mathrm{m}$, which is larger than the grid size-limited sheath thickness $\Delta_{\mathrm{sh}} \approx 0.15 \mu \mathrm{m}$. Earlier simulations ${ }^{18,24,27}$ also show that the necessary condition (2) holds (sometimes rather closely ${ }^{26}$ ) when self-injection occurs.

The background plasma is perfectly homogeneous in our simulations. However, localized density depressions may naturally occur in gas jet targets. Our relation (2) helps 
evaluate the scale of depressions necessary to bring about electron injection. The normalized bubble length, $k_{p} L_{b}$, is proportional to the driver amplitude. ${ }^{4-6}$ Even if the driver evolution is negligible (as in the case of beam-driven bubble ${ }^{1,5}$ ), the bubble necessarily expands upon crossing a density down-ramp, and electrons may be self-injected. ${ }^{45}$ If the down-ramp length is longer than a slippage distance, $L_{\text {ramp }} \gg c T_{\text {slip }}$, then the bubble evolution is slow, and Eq. (2) applies. The bubble length in the nonuniform plasma is $L_{b}(z)=\kappa\left(2 \pi / k_{p 0}\right) / \sqrt{\tilde{n}_{e}(z)}$, where the parameter $\kappa$ (determined empirically from simulations) is between 1 and 2 . We assume a power-law density profile, $\tilde{n}_{e}(z) \equiv n_{e}(z) / n_{0}=1-A\left[\left(z-z_{\text {in }}\right) / L_{\text {ramp }}\right]^{\alpha}$, where $\tilde{n}_{e}\left(z<z_{\text {in }}\right)=n_{0}$ and $\tilde{n}_{e}\left(z>z_{\text {in }}+L_{\text {ramp }}\right)=1-A<1$. To use Eq. (2), we substitute $z-z_{\text {in }}=c T_{\text {slip }}$ and find the relation between the length of the ramp $L_{\text {ramp }}$ and density depression $A$ necessary to incur injection:

$$
L_{\text {ramp }}<c T_{\text {slip }}\left[A \kappa \pi /\left(k_{p 0} \Delta_{\text {sh }}\right)\right]^{1 / \alpha} .
$$

As an example, our simulation corresponds to $c T_{\text {slip }} \approx 75 \mu \mathrm{m}$, $\kappa \approx 1.4$ and $k_{p 0} \Delta_{\mathrm{sh}} \approx 0.07$. In this situation, Eq. (3) predicts that a $10 \%$ density depression $(A=0.1)$ with a parabolic profile $(\alpha=2)$ may produce self-injection if the scale length of density variation is less than $200 \mu \mathrm{m}$. For a linear ramp, $\alpha=1$, the condition for self-injection becomes $L_{\text {ramp }}<500 \mu \mathrm{m}$.

Figure 2(e) indicates that only electrons with impact parameters such that they enter the sheath are collected and accelerated. Nearly $40 \%$ of particles from the cylindrical shell with thickness $\Delta R_{\text {coll }} \approx 2 \mu \mathrm{m}$, radius $R_{\text {coll }} \approx 8 \mu \mathrm{m}$ (which is very close to the laser spot size), and length $\Delta z_{\exp } \approx 400 \mu \mathrm{m}$ end up inside the bucket. On the other hand, first-principle 3D PIC simulation of Sec. III, while agreeing with the WAKE simulation on all dynamical features of self-injection process, shows only $0.5 \%$ collection efficiency. Evidently, correct calculation of injected charge requires fully kinetic modeling. Similar situation has been observed earlier by Morshed et $a l^{43}$ - promotion of WAKE macroparticles to the nonquasistatic, fully self-consistent macroparticles gave the injected charge consistent with full PIC simulation, whereas promotion to the nonquasistatic test electrons overestimated the charge by more than an order of magnitude. Understanding of the large discrepancy between the collection efficiency of test electrons and PIC macroparticles is the subject of our ongoing research. Meanwhile, we conclude that injection of electrons from a very narrow range of impact parameters (in agreement with earlier results ${ }^{16}$ ), together with low collection efficiency in realistic PIC modeling, makes massive self-injection during the slippage time very unlikely in rarefied plasmas $\left(\gamma_{g}>10\right)$. Injection candidates remain the minority, and their contribution to the evolution of the accelerating structure is insignificant. This justifies the quasistatic treatment of the plasma electrons making up the accelerating structure and validates the test-particle model of self-injection process.

\section{B. Self-injection into an oscillating bubble: Formation of quasi-monoenergetic collimated electron bunch}

As shown in Fig. 1, bubble expansion starts near the edge of the density plateau and continues until $z \approx 1 \mathrm{~mm}$.
Electrons reaching the highest energy are collected during this period. Contraction of the bubble between $z=1$ and $1.25 \mu \mathrm{m}$ terminates injection and expels electrons injected at the end of expansion period; a quasi-monoenergetic electron bunch forms at this stage. WAKE calculates all potentials directly, which makes the Hamiltonian analysis of test particle tracking straightforward. Using the definitions of normalized momentum $\mathbf{p} \equiv \mathbf{p} /\left(m_{e} c\right)$, wake potential $\Phi=|e|$ $\left(\varphi-A_{z}\right) /\left(m_{e} c^{2}\right)$, envelope of the laser vector potential $\mathbf{a} \equiv|e| \mathbf{a} /\left(m_{e} c^{2}\right)$, and $\gamma_{e}=\left(1+\mathbf{p}^{2}+\mathbf{a}^{2} / 2\right)^{1 / 2}$, we introduce the normalized time-averaged MF Hamiltonian $H_{\mathrm{MF}}(r, z, \xi)=\gamma_{e}+\Phi-p_{z}{ }^{24,26}$ For the quasistatic macroparticles, $H_{\mathrm{MF}} \equiv 1 .^{38}$ Test electrons (which are not assumed to be quasistatic) move in explicitly time-dependent potentials; hence, $H_{\mathrm{MF}}$ changes in the course of propagation according to $d H_{\mathrm{MF}} / d t=\partial H_{\mathrm{MF}} / \partial t$. For a test electron moving away from the bubble, $H_{\mathrm{MF}}=\gamma_{e}+\Phi-p_{z} \rightarrow \sqrt{1+\mathbf{p}^{2}}$ $-p_{z}>0$. Hence, the electron is confined inside the bucket at all times (trapped) if the $H_{\mathrm{MF}}$ remains negative in the course of interaction. As soon as the bubble stabilizes, $H_{\mathrm{MF}}$ is conserved. All test electrons can be then divided into 3 groups: (1) $H_{\mathrm{MF}}<0$-trapped; (2) $0<H_{\mathrm{MF}}<1$-injected (accelerated); and (3) $H_{\mathrm{MF}}>1$. All the three groups are represented in Figs. 3(b) and 3(d), where the plasma bubble is shown at the stationary points of full expansion and full contraction. Electron phase space for the fully expanded bubble [labeled (2) in Fig. 3(e)] shows that the bubble expansion causes a reduction in $H_{\mathrm{MF}}{ }^{24,26}$ The condition $H_{\mathrm{MF}}<1$ is thus necessary for injection and initial acceleration. For instance, it can be used for promotion of test electrons into the nonquasistatic electron beam particles in order to selfconsistently incorporate beam loading into the model. Conversely, even minimal bubble contraction may raise $H_{\mathrm{MF}}$ significantly. The inset of Fig. 3(e) shows that electrons with $0<H_{\mathrm{MF}}<2$ are accelerated as effectively as those which are formally trapped. Hence, the natural evolution of the structure may result in violation of the sufficient trapping condition; this, however, does not disrupt acceleration with good collimation and low energy spread.

When the bubble has fully expanded and subsequent contraction begins, injection stops. According to Fig. 3(f), the longitudinally nonuniform, comoving accelerating gradient changes insignificantly during the period of contraction. Figure 3(e) shows that the tail of the electron bunch, continuously exposed to the highest gradient, equalizes in energy with earlier injected electrons within an interval of $0.25 \mathrm{~mm}$. Thus, a quasi-monoenergetic bunch with the energy $E=360_{-20}^{+40} \mathrm{MeV}$ and $4.3 \mathrm{mrad}$ divergence forms long before the nonlinear dephasing limit, ${ }^{6} L_{d}=\left(\gamma_{g}^{2} / 3\right) L_{b} \approx 1.7 \mathrm{~mm}$. Therefore, limiting the plasma length to one period of bubble oscillation gives a high-quality, collimated electron beam. ${ }^{46}$

\section{Continuous self-injection caused by self- compression of the driving pulse}

Although a monoenergetic electron bunch forms early, the general experimental trend is to push the accelerator efficiency to the limit and use the entire dephasing length. Electron beam quality, however, can be compromised in this 

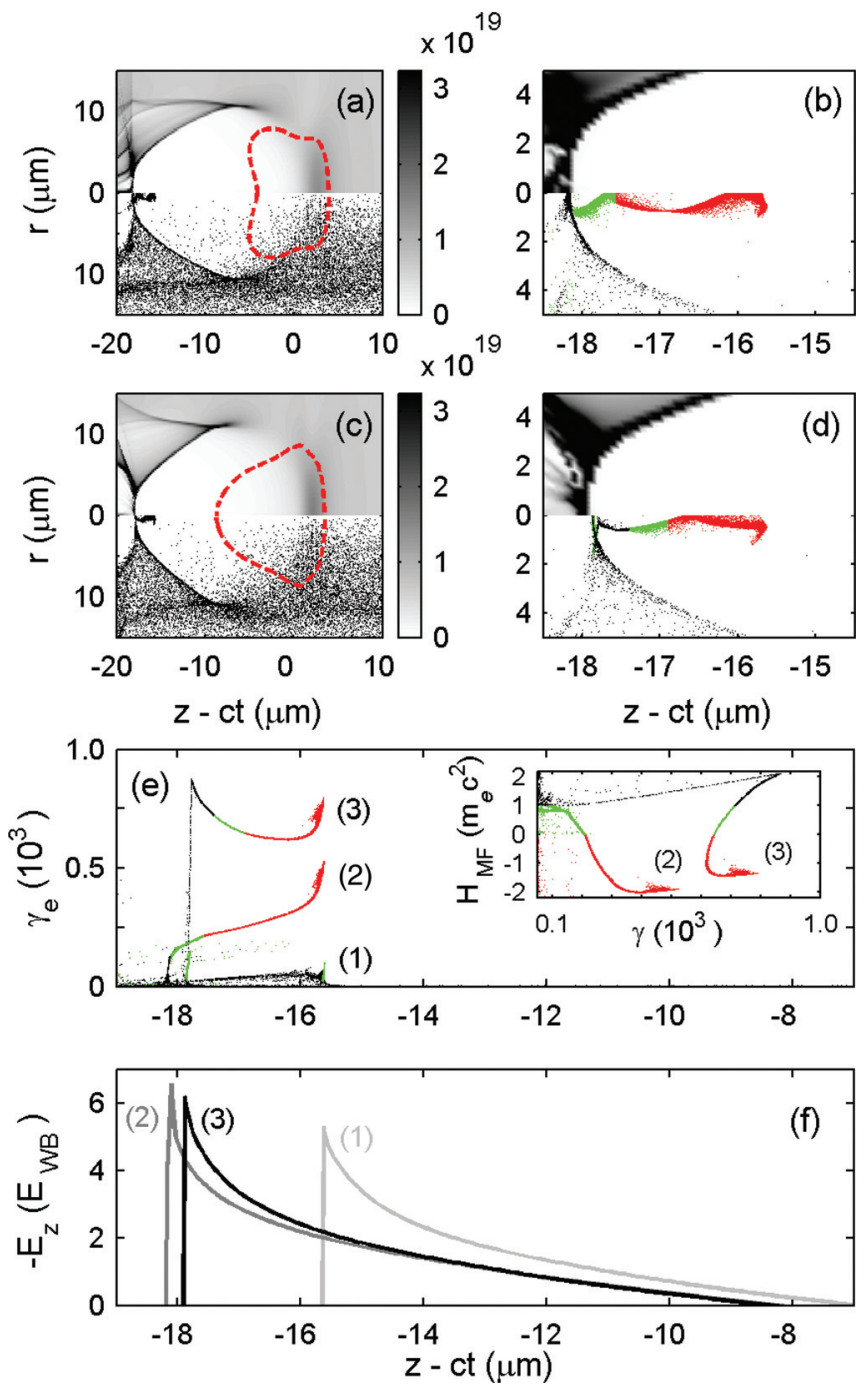

FIG. 3. (Color online) Phase space rotation and formation of a monoenergetic bunch. (a) Fully expanded bubble [cf. position (2) of Fig. 1(a)]. (c) Contracted bubble [cf. position (3) of Fig. 1(a)]. Top half: quasistatic electron density (in $\mathrm{cm}^{-3}$ ); bottom: number density of nonquasistatic test electrons. The dashed curve is a laser intensity isocontour at $\exp (-2)$ of the peak. Panels (b) and (d): blowup of the bubble rear from panels (a) and (c); test electrons are color coded according to $H_{\mathrm{MF}}<0$ [red (dark gray)], $0<H_{\mathrm{MF}}<1$ [green (light gray)], $H_{\mathrm{MF}}>1$ (black). (e) Phase space rotation of injected test electrons. Longitudinal phase space is shown at the positions (1)-(3) of Fig. 1(a). (1) Injection begins. (2) The bubble is fully expanded, injection stops, and phase space rotation begins. The bucket slightly contracts between positions (2) and (3). (3) Electrons injected lately equalize in energy with those injected earlier. Quasi-monoenergetic bunch forms. Inset shows $H_{\mathrm{MF}}$ vs energy gain for the fully expanded (2) and contracted (3) bubble. (f) Axial line-outs of the accelerating gradient (normalized to $\left.E_{W B}=m_{e} c \omega_{p e} /|e| \approx 2.5 \mathrm{GV} / \mathrm{cm}\right)$.

pursuit. The driver pulse evolves continuously, which may cause uninterrupted electron injection and emittance growth. ${ }^{12,13,17}$ Understanding the physical mechanism of continuous injection will help in controlling the beam quality by limiting continuous injection through appropriate choice of experimental geometry ${ }^{27,46}$ or through manipulating the phase and envelope of driving pulse.

Running the simulation until the nonlinear dephasing limit, we find two distinct stages of the system evolution. Stage I (studied above) corresponds to a single oscillation of the laser spot size and produces a monoenergetic electron
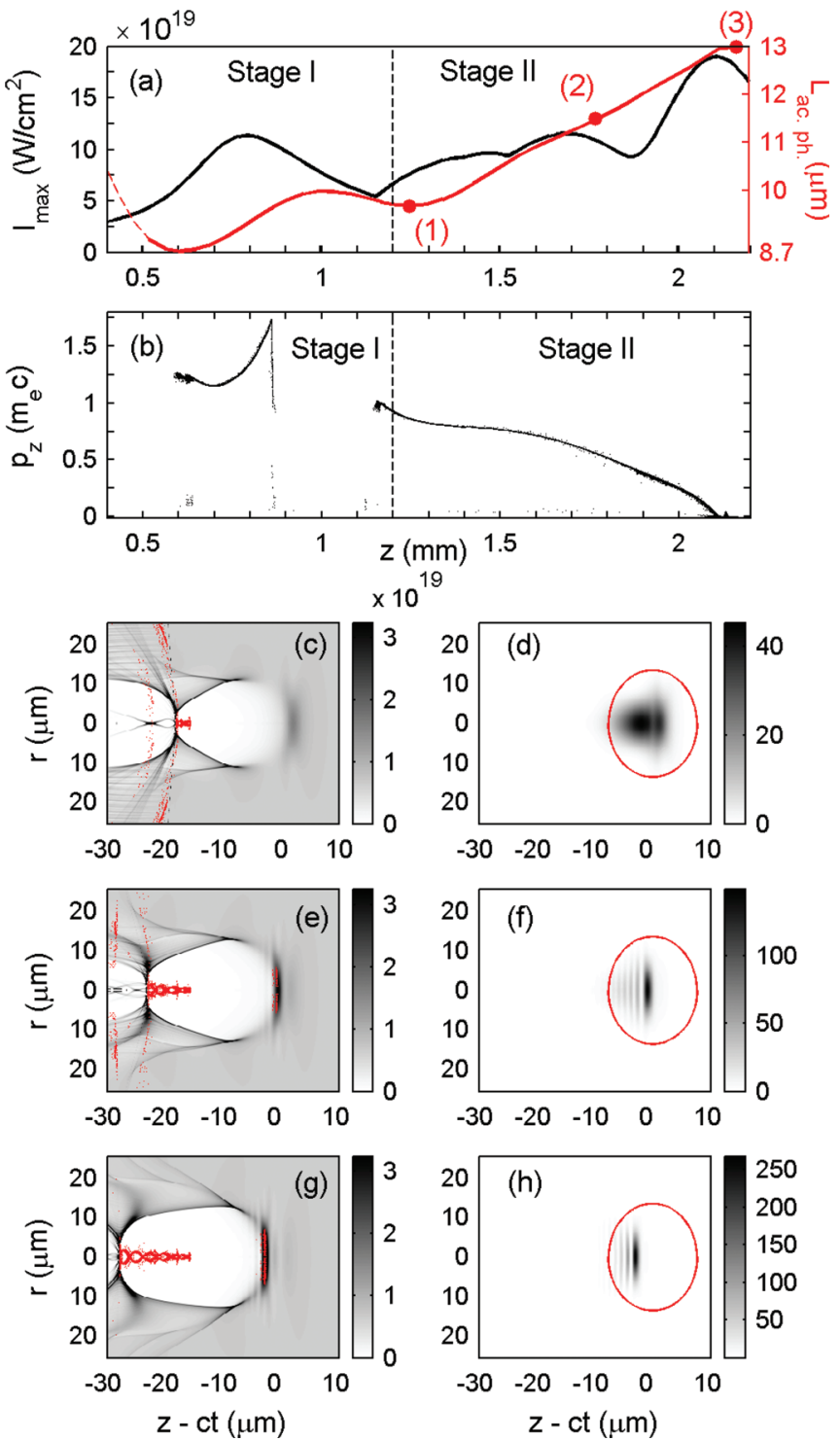

FIG. 4. (Color online) Pulse self-compression and continuous injection. (a) Peak laser intensity (black) and the length of the accelerating phase vs propagation length [red (gray)]. (b) Energy of test electrons versus their initial positions at $z=2.2 \mathrm{~mm}$. The leading quasi-monoenergetic electron beam forms during stage I (one period of bubble size oscillation). Continuous bubble expansion during stage II causes continuous injection with broad energy spectrum. Panels (c), (e), and (g) show electron density (in $\mathrm{cm}^{-3}$ ) and test electrons with $\gamma>\gamma_{g}$ [red (gray) dots]. Panels (d), (f), and (h): normalized laser intensity $|a|^{2}$; red (gray) line: isocontour of an incident pulse intensity at $\exp (-2)$ of the peak. Panels (c) and (d); (e) and (f); and (g) and (h) correspond to the positions (1), (2), and (3) of panel (a), respectively. Contraction of the driver pulse (formation of a relativistic piston) causes elongation of the bubble and continuous injection.

bunch. Stage II is characterized by a gradual increase of laser intensity (up to $2 \times 10^{20} \mathrm{~W} / \mathrm{cm}^{2}$ ) and steady elongation of the bubble. Figure 4 shows that the bubble elongation is accompanied by continuous injection and growth of the energy spread. At the end of the run, the number of continuously injected test electrons $\left(\gamma>\gamma_{g}\right)$ is factor of 7.5 larger than the number of electrons in the leading quasi-monoenergetic bunch. Similar development of self-injection process has been reported elsewhere. ${ }^{12,13}$ Figures $4(\mathrm{c})-4(\mathrm{~h})$ show that the bubble growth is accompanied by self-compression of the driver pulse from 25 to roughly 5.5 fs. The compressed pulse 

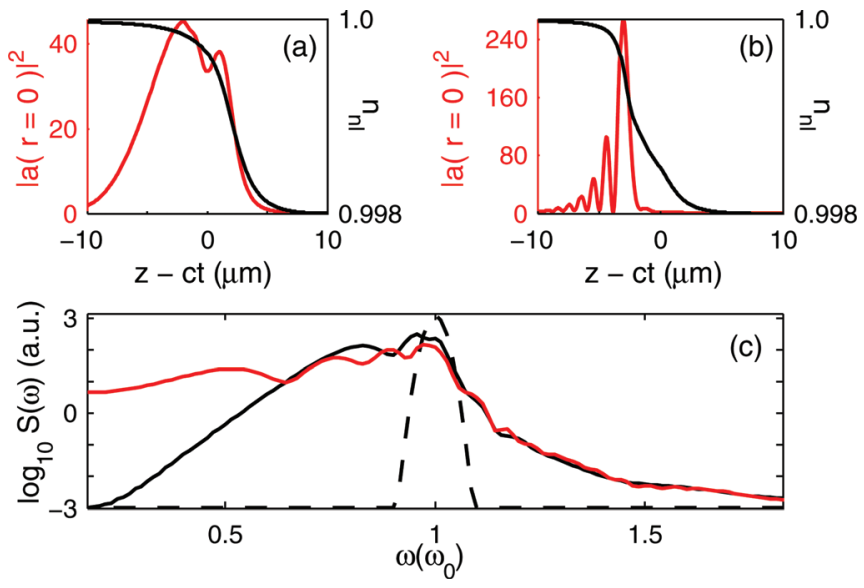

FIG. 5. (Color online) Pulse redshifting and formation of the relativistic piston. (a) Axial lineouts of normalized intensity [red (gray)] and nonlinear refractive index (black) at the position (1) of Fig. 4(a). (b) Same for the position (3) of Fig. 4(a). (c) Laser frequency spectra. Solid black corresponds to panel (a), red (gray) - to panel (b), dashed black - to the incident pulse. Strong redshifting and spectral broadening are partly caused by the comoving nonlinear index gradient. Panel (b) shows that the spectrally broadened pulse is compressed to approximately two cycles.

acts as a snow-plow; the ponderomotive push of its front preaccelerates plasma electrons to $\gamma>\gamma_{g}$ and, as is seen in Fig. $4(\mathrm{~g})$, creates a strongly compressed electron slab, $n_{e} \approx 10 n_{0}$. As a result, the electric field due to charge separation immediately behind the piston driver [Fig. 4(h)] is a factor 2.25 higher than in the case of smooth driver [Fig. 4(d)]. Behind the piston, sheath electrons receive a large kick in the backward direction and quickly become relativistic, $p_{z}$ $\approx-1.65 m_{e} c$ (in contrast to $-0.55 m_{e} c$ in the smooth driver case). As the focusing gradient is almost the same in both cases, it takes a longer time for sheath electrons to reach the axis in the piston case, which explains the bubble elongation.

Pulse self-steepening is partly caused by depletion due to wake excitation $(\sim 40 \%$ at $z=2.2 \mathrm{~mm})$ and is partly a nonlinear optical effect. ${ }^{31-33}$ Figures $5(a)$ and 5(b) show axial lineouts of normalized intensity and of the nonlinear index of refraction. The pulse leading edge witnesses the index downramp at all times. The laser frequency redshifts in the region of index gradient. At the same time, the tail traveling inside the bubble remains unshifted. Figure 5(c) shows that the laser spectrum broadens toward $\omega=\omega_{\text {pe }}$; envelope oscillations in Fig. 5(b) result from the strong reduction of the pulse central frequency. The large bandwidth explains pulse compression to roughly two cycles. In the absence of strong depletion, the compression yields the extremely high intensity of the resulting relativistic piston. The redshift of central frequency together with the front steepening additionally slow down the pulse and the bubble and provide another reason for the occurrence of continuous injection. Any way to compensate the nonlinear chirp in the pulse front will help delay pulse contraction and slow-down, partially suppressing continuous injection.

\section{VALIDATION OF SELF-INJECTION SCENARIOS IN FIRST-PRINCIPLE 3D PIC SIMULATION}

Collective fields of the electron beam, neglected in the test-particle treatment, are known to change the shape of the sheath and, thus, reduce accelerating gradient, eventually terminating self-injection. ${ }^{35}$ In this section, we verify the testparticle results by running a fully explicit 3D PIC simulation with the identical set of initial conditions. We use the quasicylindrical code CALDER-CIRC, ${ }^{40}$ which preserves realistic geometry of interaction and accounts for the axial asymmetry by decomposing electromagnetic fields (laser and wake) into a set of poloidal modes (whereas the particles remain in full 3D). Well preserved cylindrical symmetry during the interaction enables us to use just the two lowest order modes and thus reduce $3 \mathrm{D}$ problem to an essentially $2 \mathrm{D}$ one. We suppress the sampling noise by using large number of macroparticles (45 per cell) and high resolution in the direction of propagation, $d z=0.125 c / \omega_{0}$. The aspect ratio is $d r / d z$ $=15.6$ and the time step $d t=0.1244 \omega_{0}^{-1}$. Figure 6 shows that despite a much coarser grid, larger time step, and underlying approximations, the WAKE simulation correctly captures all relevant physics of plasma wake evolution and dynamics of electron self-injection. In addition, CALDER-CIRC having fully self-consistent macroparticle dynamics yields the complete electron phase space and, thus, calculates precisely injected charge and beam emittance.

In spite of great difference in the algorithms and physics content, both codes demonstrate the same correlation between the laser and bubble evolution. Self-injection begins, terminates, and resumes at exactly the same positions along the propagation axis in both runs. Figures $6(\mathrm{a}), 6(\mathrm{~d})$, and $6(\mathrm{~g})$ show the result of stage I-formation of quasi-monoenergetic electron bunch before dephasing. Self-fields of the bunch are unable to prevent the bucket contraction and partial detrapping of electrons. The bunch phase space has a characteristic "U"-shape produced by the phase space rotation. The bunch has $8 \%$ energy spread around $245 \mathrm{MeV}$ and a charge $Q_{\text {mono }} \approx 230 \mathrm{pC}$, which is consistent with the earlier 3D PIC simulations $^{24}$ [in addition, electrons from the second bucket produce a separate, rather diffuse peak around $150 \mathrm{MeV}$ in Fig. 6(d)]. The bunch duration, $t_{b}=10 \mathrm{fs}$, is the same as in the test-particle simulation; whereas divergence, $13 \mathrm{mrad}$, is three times higher. The normalized transverse emittance, $\varepsilon_{N, y} \approx \varepsilon_{N, x}=\left(m_{e} c\right)^{-1}\left(\left\langle\Delta x^{2}\right\rangle\left\langle\Delta p_{x}^{2}\right\rangle-\left\langle\Delta x \Delta p_{x}\right\rangle^{2}\right)^{1 / 2} \approx 8.5 \pi \mathrm{mm}$ $\mathrm{mrad}$, appears to be a factor of 15 larger than that obtained in the test-particle simulation. Hence, charge and current density deposition in full PIC model are essential for precise calculation of the phase space volume of self-injected electrons.

The difference between the phase spaces of WAKE test electrons and CALDER-CIRC macroparticles, clearly seen in Fig. 6(g), can be attributed to the effect of beam loading, which reduces the accelerating gradient along the bunch and slows down phase space rotation, ultimately reducing the bunch energy by $30 \%$. To evaluate the contribution of beam loading, we approximate the bunch density as a flat-top distribution with a Gaussian radial profile, $n_{b}(r)=n_{b 0} \exp$ $\left(-r^{2} / \sigma_{b}^{2}\right)$, where $\sigma_{b} \approx 1.25 \mu \mathrm{m}$ and $n_{b 0}=\left(Q_{\text {mono }} /|e|\right)$ $\left(\pi c t_{b} \sigma_{b}^{2}\right)^{-1} \approx 10^{20} \mathrm{~cm}^{-3}$. According to Ref. 35, the sheath electrons cross the axis, and the bubble remains closed if $K \equiv R_{\mathrm{b}}^{4} /\left(8 r_{\mathrm{t}}^{2} \Lambda_{0}\right)>1$, where $\Lambda_{0}=\left(\sigma_{b}^{2} / 2\right)\left(n_{b 0} / n_{0}\right)$ is the normalized charge per unit length, $r_{\mathrm{t}}$ is the bubble radius in the transverse cross-section taken at the front tip of the bunch, and $R_{\mathrm{b}}$ is the bubble radius in the central cross-section. 

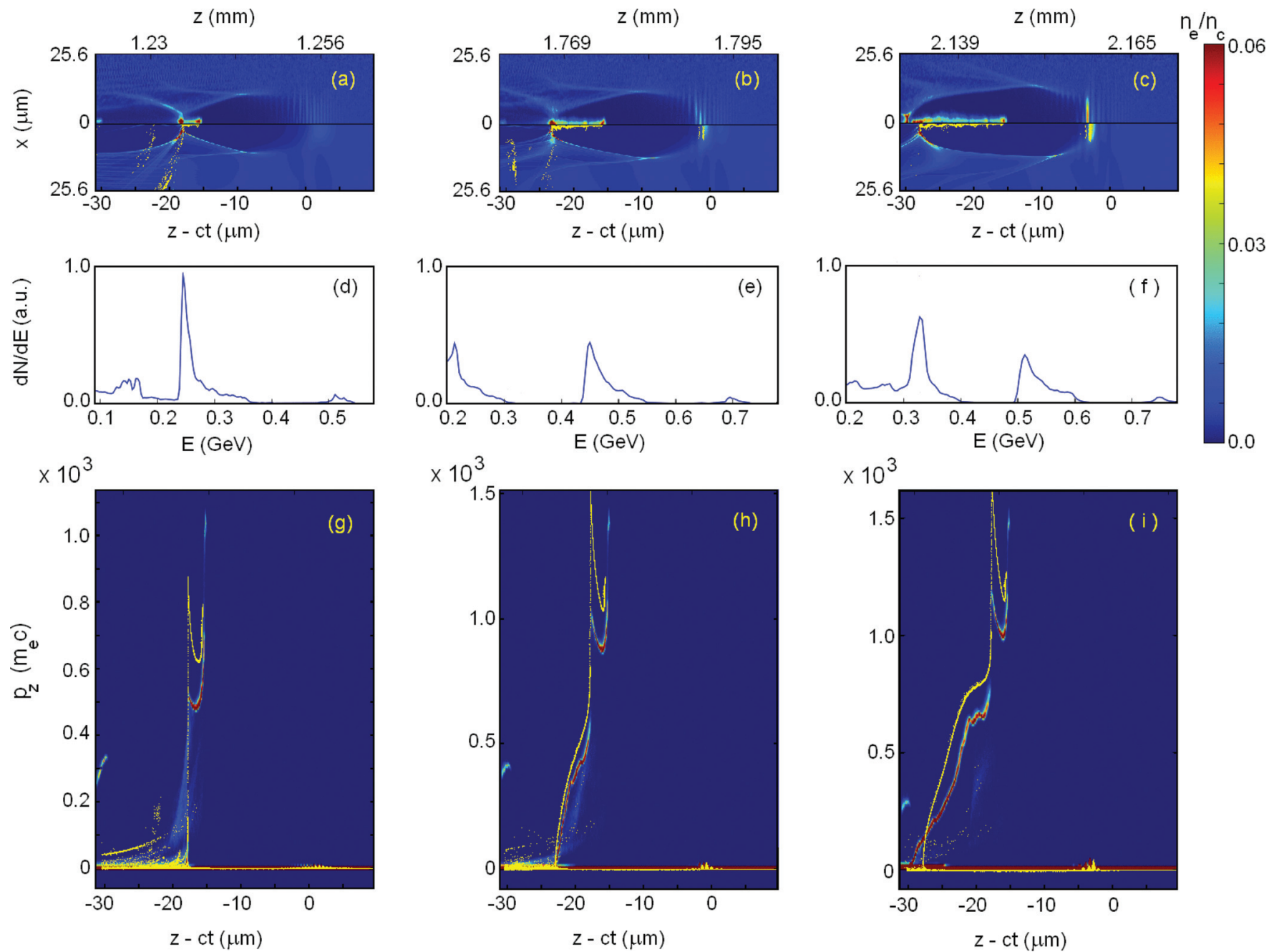

FIG. 6. (Color) Continuous injection in quasistatic (wAKE with test particles) and full 3D PIC (CALDER-CIRC) simulations. (a)-(c) Electron density from CALDERCIRC (top half) and WAKE (bottom) runs. Yellow dots are the test electrons with $\gamma>\gamma_{g}$. (d)-(f) Electron energy spectrum (CALDER-CIRC). (g)-(i) Longitudinal phase space (colormap—CALDER-CIRC; WAKE test electrons-yellow dots). Panels (a), (b), and (c) are counterparts of Figs. 4(c), 4(e), and 4(g).

Examination of Fig. 6(a) gives $R_{\mathrm{b}} \approx 2 r_{\mathrm{t}} \approx 10 \mu \mathrm{m}$, and $K \approx 4.3$. This explains the relatively mild beam loading effect during stage I.

Figures 6(a)-6(c) show that continuous injection developed in both CALDER-CIRC and WAKE runs in exactly the same fashion. Continuously injected charge in CALDER-CIRC simulation reaches $Q_{\text {cont }} \approx 1.5 \mathrm{nC}$ near the dephasing limit; the beam divergence is $35.6 \mathrm{mrad}$, and normalized transverse emittances are $35 \pi \mathrm{mm}$ mrad. The ratio $Q_{\text {cont }} / Q_{\text {mono }} \approx 6.5$ is close to the test particle result of Sec. II C. In spite of the large amount of injected charge, the bubble shape at the dephasing point is almost unaffected by the presence of the electron beam; this rules out beam loading as a cause of continuous injection. Analytical estimate confirms that the bucket is still not fully loaded $(K \approx 1.5)$ and injection in the CALDER-CIRC simulation continues beyond the dephasing point. Therefore, apart from slight reduction of the accelerating gradient, beam loading brings no new physical features into the scenario of continuous injection discussed in section II C. Continuous injection can be thus associated solely with frequency redshift and self-compression of the driver pulse.

\section{CONCLUSIONS}

A time-varying electron density bubble created by the radiation pressure of a tightly focused laser pulse guides the pulse through a uniform, rarefied plasma, traps ambient plasma electrons, and accelerates them to gigaelectron voltlevel energy. Natural pulse evolution (nonlinear focusing and self-compression) is in most cases sufficient to initiate and terminate self-injection. The bubble dynamics and the self-injection process are governed primarily by driver evolution. Expansion of the bubble facilitates injection, whereas stabilization and contraction extinguishes injection and suppresses low-energy background. Simultaneously, longitudinal nonuniformity of the accelerating gradient causes rapid phase space rotation. Although beam loading reduces the accelerating gradient and slows down phase space rotation, a quasi-monoenergetic, well collimated electron bunch forms long before dephasing. Phase self-modulation and frequency redshift due to the wake excitation cause gradual compression of the driver pulse. In turn, the formation of a few-cycle duration, strongly relativistic piston driver causes bubble elongation and continuous secondary injection. A combination of reduced and fully 
self-consistent 3D PIC simulations shows that the role of beam loading in the early stage of continuous injection is insignificant. In our subsequent publications, we shall demonstrate how the appropriate shaping of initial amplitude and phase of the driver pulse helps to control continuous injection and improve the final beam quality.

The reported results highlight the importance of reduced physics models. Reduced models not only lower the computational cost of simulations (sometimes by many orders of magnitude) but also allow for the identification of the underlying physical processes responsible for the observed phenomena. The self-injection dynamics and its relation to the nonlinear optical evolution of the driver were understood using especially simple simulation tools (cylindrical quasistatic PIC code with fully $3 \mathrm{D}$ dynamic test particle module). In practical terms, this means that the system performance (electron beam duration, mean energy, energy spread, and, very roughly, divergence) can be approximately assessed without recourse to computationally intensive 3D PIC simulations. It appears that, however, calculation of the beam charge and transverse emittance still needs a 3D fully kinetic simulation. Clarifying the nature of subtle self-consistent effects affecting the phase space volume of self-injected electrons in various numerical models and establishing the true physical origin of these effects are the subject of our further work.

\section{ACKNOWLEDGMENTS}

The work is partly supported by the U.S. Department of Energy Grant Nos. DE-FG02-96-ER-40954, DE-FG0207ER54945, and DE-FG02-08ER55000. S.Y.K., B.A.S., and D.P.U. acknowledge the support of the Chemical Sciences, Geosciences and Biosciences Division, Office of Basic Energy Sciences, Office of Science, the U.S. Department of Energy, and DARPA. A.B. and E.L. acknowledge the support of LASERLAB-EUROPE/LAPTECH through EC FP7 Contract No. 228334. The authors acknowledge Centre de Calcul pour la Recherche et la Technologie at CEA for providing high-performance computing resources.

${ }^{1}$ J. B. Rosenzweig, B. Breizman, T. Katsouleas, and J. J. Su, Phys. Rev. A 44, R6189 (1991).

${ }^{2}$ P. Mora and T. M. Antonsen, Jr., Phys. Rev. E 53, R2068 (1996).

${ }^{3}$ A. Pukhov and J. Meyer-ter-Vehn, Appl. Phys. B: Lasers Opt. 74, 355 (2002).

${ }^{4}$ S. Gordienko and A. Pukhov, Phys. Plasmas 12, 043109 (2005).

${ }^{5}$ W. Lu, C. Huang, M. Zhou, M. Tzoufras, F. S. Tsung, W. B. Mori, and T. Katsouleas, Phys. Plasmas 13, 056709 (2006).

${ }^{6}$ W. Lu, M. Tzoufras, C. Joshi, F. S. Tsung, W. B. Mori, J. Vieira, R. A. Fonseca, and L. O. Silva, Phys. Rev. ST Accel. Beams 10, 061301 (2007).

${ }^{7}$ A. G. R. Thomas, Phys. Plasmas 17, 056708 (2010).

${ }^{8}$ S. P. D. Mangles, C. D. Murphy, Z. Najmudin, A. G. R. Thomas, J. L. Collier, A. E. Dangor, E. J. Divall, P. S. Foster, J. G. Gallacher, C. J. Hooker, D. A. Jaroszynski, A. J. Langley, W. B. Mori, P. A. Norreys, F. S. Tsung, R. Viskup, B. R. Walton, and K. Krushelnick, Nature (London) 431, 535 (2004).

${ }^{9}$ C. G. D. Geddes, Cs. Toth, J. van Tilborg, E. Esarey, C. B. Schroeder, D. Bruhwiler, C. Nieter, J. Cary, and W. P. Leemans, Nature (London) 431, 538 (2004).

${ }^{10}$ J. Faure, Y. Glinec, A. Pukhov, S. Kiselev, S. Gordienko, E. Lefebvre, J.P. Rousseau, F. Burgy, and V. Malka, Nature (London) 431, 541 (2004).

${ }^{11}$ W. P. Leemans, B. Nagler, A. J. Gonsalves, Cs. Tóth, K. Nakamura, C. G. R. Geddes, E. Esarey, C. B. Schroeder, and S. M. Hooker, Nat. Phys. 2, 696 (2006).
${ }^{12}$ S. Kneip, S. R. Nagel, S. F. Martins, S. P. D. Mangles, C. Bellei, O. Chekhlov, R. J. Clarke, N. Delerue, E. J. Divall, G. Doucas, K. Ertel, F. Fiúza, R. Fonseca, P. Foster, S. J. Hawkes, C. J. Hooker, K. Krushelnick, W. B. Mori, C. A. J. Palmer, K. Ta Phuoc, P. P. Rajeev, J. Schreiber, M. J. V. Streeter, D. Urner, J. Vieira, L. O. Silva, and Z. Najmudin, Phys. Rev. Lett. 103, 035002 (2009).

${ }^{13}$ D. H. Froula, C. E. Clayton, T. Döppner, K. A. Marsh, C. P. J. Barty, L. Divol, R. A. Fonseca, S. H. Glenzer, C. Joshi, W. Lu, S. F. Martins, P. Michel, W. B. Mori, J. P. Palastro, B. B. Pollock, A. Pak, J. E. Ralph, J. S. Ross, C. W. Siders, L. O. Silva, and T. Wang, Phys. Rev. Lett. 103, 215006 (2009).

${ }^{14}$ J. E. Ralph, C. E. Clayton, F. Albert, B. B. Pollock, S. F. Martins, A. E. Pak, K. A. Marsh, J. L. Shaw, A. Till, J. P. Palastro, W. Lu, S. H. Glenzer, L. O. Silva, W. B. Mori, C. Joshi, and D. H. Froula, Phys. Plasmas 17, 056709 (2010).

${ }^{15}$ C. E. Clayton, J. E. Ralph, F. Albert, R. A. Fonseca, S. H. Glenzer, C. Joshi, W. Lu, K. A. Marsh, S. F. Martins, W. B. Mori, A. Pak, F. S. Tsung, B. B. Pollock, J. S. Ross, L. O. Silva, and D. H. Froula, Phys. Rev. Lett. 105, 105003 (2010).

${ }^{16}$ F. S. Tsung, W. Lu, M. Tzoufras, W. B. Mori, C. Joshi, J. M. Vieira, L. O. Silva, and R. A. Fonseca, Phys. Plasmas 13, 056708 (2006).

${ }^{17}$ S. F. Martins, R. A. Fonseca, W. Lu, W. B. Mori, and L. O. Silva, Nat. Phys. 6, 311 (2010).

${ }^{18}$ S. Y. Kalmykov, S. A. Yi, A. Beck, A. F. Lifschitz, X. Davoine, E. Lefebvre, A. Pukhov, V. Khudik, G. Shvets, S. A. Reed, P. Dong, X. Wang, D. Du, S. Bedacht, R. Zgadzaj, W. Henderson, A. Bernstein, G. Dyer, M. Martinez, E. Gaul, T. Ditmire, and M. C. Downer, New J. Phys. 12, 045019 (2010).

${ }^{19}$ P. Dong, S. A. Reed, S. A. Yi, S. Kalmykov, G. Shvets, M. C. Downer, N. H. Matlis, W. P. Leemans, C. McGuffey, S. S. Bulanov, V. Chvykov, G. Kalintchenko, K. Krushelnick, A. Maksimchuk, T. Matsuoka, A. G. R. Thomas, and V. Yanovsky, Phys. Rev. Lett. 104, 134801 (2010).

${ }^{20}$ M. H. Helle, D. Kaganovich, D. F. Gordon, and A. Ting, Phys. Rev. Lett. 105, 105001 (2010).

${ }^{21}$ H. Xu, W. Yu, P. Lu, V. K. Senecha, F. He, B. Shen, L. Qian, R. Li, and Z. Xu, Phys. Plasmas 12, 013105 (2005).

${ }^{22}$ A. Oguchi, A. Zhidkov, K. Takano, E. Hotta, K. Nemoto, and K. Nakajima, Phys. Plasmas 15, 043102 (2008).

${ }^{23}$ A. Zhidkov, T. Fujii, and K. Nemoto, Phys. Rev. E 78, 036406 (2008).

${ }^{24}$ S. Kalmykov, S. A. Yi, V. Khudik, and G. Shvets, Phys. Rev. Lett. 103, 135004 (2009).

${ }^{25}$ A. Zhidkov, J. Koga, T. Hosokai, T. Fujii, Y. Oishi, K. Nemoto, and R. Kodama, Phys. Plasmas 17, 083101 (2010).

${ }^{26}$ S. Y. Kalmykov, A. Beck, S. A. Yi, V. Khudik, B. A. Shadwick, E. Lefebvre, and M. C. Downer, AIP Conf. Proc. 1299, 174 (2010).

${ }^{27}$ S. Y. Kalmykov, S. A. Yi, A. Beck, A. F. Lifschitz, X. Davoine, E. Lefebvre, V. Khudik, G. Shvets, and M. C. Downer, Plasma Phys. Controlled Fusion 53, 014006 (2011).

${ }^{28}$ S. P. D. Mangles, A. G. R. Thomas, O. Lundh, F. Lindau, M. C. Kaluza, A. Persson, C.-G. Wahlström, K. Krushelnick, and Z. Najmudin, Phys. Plasmas 14, 056702 (2007).

${ }^{29}$ J. Osterhoff, A. Popp, Zs. Major, B. Marx, T. P. Rowlands-Rees, M. Fuchs, M. Geissler, R. Hörlein, B. Hidding, S. Becker, E. A. Peralta, U. Schramm, F. Grüner, D. Habs, F. Krausz, S. M. Hooker, and S. Karsch, Phys. Rev. Lett. 101, 085002 (2008).

${ }^{30}$ G. Z. Sun, E. Ott, Y. C. Lee, and P. Guzdar, Phys. Fluids 30, 526 (1987).

${ }^{31}$ J. Faure, Y. Glinec, J. J. Santos, F. Ewald, J.-P. Rousseau, S. Kiselev, A. Pukhov, T. Hosokai, and V. Malka, Phys. Rev. Lett. 95, 205003 (2005).

${ }^{32}$ C.-H. Pai, Y.-Y. Chang, L.-C. Ha, Z.-H. Xie, M.-W. Lin, J.-M. Lin, Y.-M. Chen, G. Tsaur, H.-H. Chu, S.-H. Chen, J.-Y. Lin, J. Wang, and S.-Y. Chen, Phys. Rev. A 82, 063804 (2010).

${ }^{33}$ J. Vieira, F. Fiúza, L. O. Silva, M. Tzoufras, and W. B. Mori, New. J. Phys. 12, 045025 (2010).

${ }^{34}$ C. D. Decker, W. B. Mori, K.-C. Tzeng, and T. Katsouleas, Phys. Plasmas 3, 2047 (1996).

${ }^{35}$ M. Tzoufras, W. Lu, F. S. Tsung, C. Huang, W. B. Mori, T. Katsouleas, J. Vieira, R. A. Fonseca, and L. O. Silva, Phys. Plasmas 16, 056705 (2009).

${ }^{36}$ F. Grüner, S. Becker, U. Schramm, T. Eichner, M. Fuchs, R. Weingartner, D. Habs, J. Meyer-ter-Vehn, M. Geissler, M. Ferrario, L. Serafini, B. van der Geer, H. Backe, W. Lauth, and S. Reiche, Appl. Phys. B: Lasers Opt. 86, 431 (2007).

${ }^{37}$ F. V. Hartemann, D. J. Gibson, W. J. Brown, A. Rousse, K. Ta Phuoc, V. Malka, J. Faure, and A. Pukhov, Phys. Rev. ST Accel. Beams 10, 011301 (2007). 
${ }^{38}$ P. Mora and T. M. Antonsen, Jr., Phys. Plasmas 4, 217 (1997).

${ }^{39}$ V. Malka, J. Faure, J. R. Marques, F. Amiranoff, J. P. Rousseau, S. Ranc, J. P. Chambaret, Z. Najmudin, B. Walton, P. Mora, and A. Solodov, Phys. Plasmas 8, 2605 (2001).

${ }^{40}$ A. F. Lifschitz, X. Davoine, E. Lefebvre J. Faure, C. Rechatin, and V. Malka, J. Comput. Phys. 228, 1803 (2009).

${ }^{41}$ V. Ramanathan, S. Banerjee, N. Powers, N. Cunningham, N. A. ChandlerSmith, Kun Zhao, K. Brown, D. Umstadter, S. Clarke, S. Pozzi, J. Beene, C. R. Vane, and D. Schultz, Phys. Rev. ST Accel. Beams 13, 104701 (2010).
${ }^{42}$ B. Quesnel and P. Mora, Phys. Rev. E 58, 3719 (1998).

${ }^{43}$ S. Morshed, T. M. Antonsen, Jr., and J. P. Palastro, Phys. Plasmas 17, 063106 (2010).

${ }^{44}$ D. Kaganovich, D. F. Gordon, and A. Ting, Phys. Rev. Lett. 100, 215002 (2008).

${ }^{45}$ R. G. Hemker, N. M. Hafz, and M. Uesaka, Phys. Rev. ST Accel. Beams 5, 041301 (2002).

${ }^{46}$ N. A. M. Hafz, S. K. Lee, T. M. Jeong, and J. Lee, "Evolution of selfinjected quasi-monoenergetic electron beams in a plasma bubble," Nucl. Instrum. Methods in Phys. Res. A (to be published). 